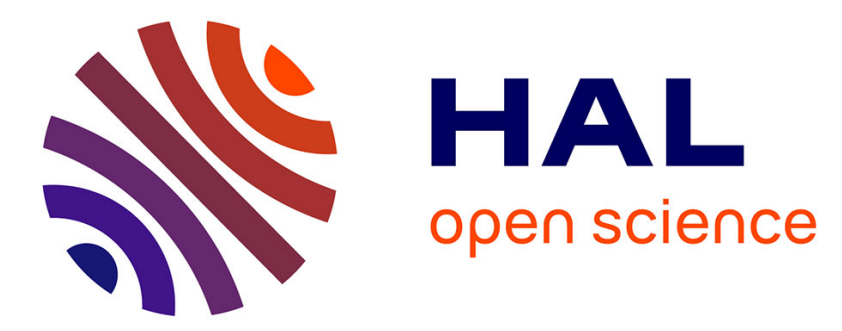

\title{
Some remarks on vehicle following control systems with delays
}

\author{
Woihida Aggoune, Dorin Şendrescu, Silviu-Iulian Niculescu
}

\section{To cite this version:}

Woihida Aggoune, Dorin Şendrescu, Silviu-Iulian Niculescu. Some remarks on vehicle following control systems with delays. 7th IFAC Workshop on Time Delay Systems TDS 2007, Sep 2007, Nantes, France. pp.103-110, 10.1016/S1474-6670(17)69271-1 . hal-02293024

\section{HAL Id: hal-02293024 \\ https://hal.science/hal-02293024}

Submitted on 20 Sep 2019

HAL is a multi-disciplinary open access archive for the deposit and dissemination of scientific research documents, whether they are published or not. The documents may come from teaching and research institutions in France or abroad, or from public or private research centers.
L'archive ouverte pluridisciplinaire HAL, est destinée au dépôt et à la diffusion de documents scientifiques de niveau recherche, publiés ou non, émanant des établissements d'enseignement et de recherche français ou étrangers, des laboratoires publics ou privés. 


\title{
SOME REMARKS ON VEHICLE FOLLOWING CONTROL SYSTEMS WITH DELAYS
}

\author{
Woihida Aggoune* Dorin Şendrescu ${ }^{* *}$ \\ Silviu-Iulian Niculescu ${ }^{* * *}$ \\ * Equipe Commande des Systèmes (ECS), ENSEA, \\ 6 Av. du Ponceau, 95014 Cergy-Pontoise Cedex, France. \\ E-mail: aggounedensea.fr \\ ** Department of Automation Control, University of Craiova, \\ Romania. \\ *** Laboratoire des Signaux et Systèmes (L2S), CNRS-Supélec \\ 3 rue Joliot-Curie, 91190 Gif-sur-Yvette, France, \\ E-mail: Silviu.Niculescullss.supelec.fr
}

\begin{abstract}
In this paper, we consider the problem of vehicle following control with delay. To solve the problem of traffic congestion, one of the solutions to be considered consists in organizing the traffic into platoons, that is groups of vehicles including a leader and a number of followers "tightly" spaced, all moving in a longitudinal direction. Excepting the stability of individual cars, the problem of avoidance of slinky type effects will be explicitly discussed. Sufficient conditions on the set of control parameters to avoid such a phenomenon will be explicitly derived in a frequency-domain setting. (c)2007 IFAC.
\end{abstract}

\section{INTRODUCTION}

Traffic congestion (irregular flow of traffic) became an important problem in the last decade mainly to the exponential increasing of the transportation around medium- and large-size cities. One of the ideas to help solving this problem was the use of automatic control to replace human drivers and their low-predictable reaction with respect to traffic problems. As an example, human drivers have reaction time between $0.25-1.25$ $\mathrm{sec}$ of around $30 \mathrm{~m}$ or more at $60 \mathrm{kms} /$ hour (see, for instance, [Sipahi and Niculescu (2007)] for a complete description of human drivers reactions, and further comments on existing traffic flow models).

A way to solve this problem is to organize the traffic into platoons, consisting in groups of vehicles including a leader and a number of followers in a longitudinal direction. In this case, the controller of each vehicle of a platoon would use the sensor information to try to reach the speed and acceleration of the preceding vehicle. Another problem to be considered is the so-called slinky-type effect (see, e.g. [Burnham et al. (1974)], [Ioannou and Chien (1993)], [Shiekholslam and Desoer (1993)] and the references therein). This is a phenomenon of amplification of the spacing errors between subsequent vehicles as vehicle index increases.

In [Huang and Ren (1998)], a control scheme to solve this multi-objective control problem was proposed. Known as autonomous intelligent cruise control, the controller in this scheme has access only to the relative state information of the preceding vehicle. This study is made under the assumptions that the lead vehicle performs a maneuver in finite time before reaching a steady state, and that prior to a maneuver, all the vehicles move at the same steady speed. The stability analysis of the system in closed-loop was performed by using a Lyapunov-Razumikhin approach leading to conservative conditions. The slinky-effect type phenomenon was discussed and some sufficient conditions to avoid slinky effects have been proposed, but without any explicit attempt in computing the 
whole set of controller's parameters guaranteeing the requested property. To the best of the authors' knowledge, such a problem has not received a definitive answer.

The aim of this paper is to give better answers to the problem mentioned above - construction of explicit control laws guaranteeing simultaneously individual stability and the avoidance of the slinky-type effect phenomenon. We use a frequency-domain method to give necessary and sufficient conditions for the individual stability analysis by computing the explicit delay bounds guaranteeing asymptotic stability. Next, we shall explicitly compute bounds on the controller's gains ensuring the avoidance of the slinky effects.

The remaining paper is organized as follows: In Section 2, the problem formulation is presented. In Section 3, we state and prove our main results. In section 4, two illustrative examples are presented. Finally, some concluding remarks end the paper.

\section{SYSTEM MODEL AND PROBLEM FORMULATION}

The general schema of a platoon of $n$ vehicles is represented below, where $x_{i}(t)$ is the position of the $i$ th vehicle with respect to some reference point $O$ and $H_{i}$ is the minimum separation distance allowable between the corresponding vehicles.

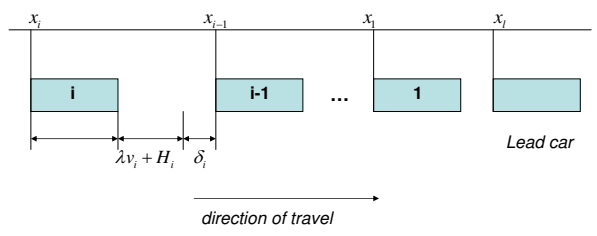

Fig. 1. Platoon configuration

The goal is to maintain a distance $\lambda v_{i}+H_{i}$ between vehicle $i$ and $i-1$, where $\lambda$ is a prescribed headway constant and $v_{i}$ the corresponding velocity (see [Huang and Ren (1998)]). The spacing error $\delta_{i}$ between the $i$ th and $(i-1)$ st vehicles is defined as :

$$
\delta_{i}(t)=x_{i-1}(t)-x_{i}(t)-\left(\lambda v_{i}+H_{i}\right)
$$

in the case of system (1).

\subsection{Model of vehicle dynamics}

For each vehicle of the platoon, the model is of the form:

$$
\left\{\begin{array}{l}
\dot{x}_{i}(t)=v_{i}(t) \\
\dot{v}_{i}(t)=\gamma_{i}(t) \\
\dot{\gamma}_{i}(t)=-\frac{1}{\eta} \gamma_{i}(t)+\frac{1}{m \eta} u_{i}\left(t-\tau_{i}\right)-\frac{1}{m \eta} T_{L},
\end{array}\right.
$$

where $x_{i}(t), v_{i}(t)$ and $\gamma_{i}(t)$ represent respectively the position, the speed and the acceleration of the $i$ th vehicle. Here, $\eta$ is the vehicle's engine time-constant, $m$ is the vehicle mass, $T_{L}$ is the load torque on the engine speed, gear ratio, grade change etc., and it is assumed to be constant. $\tau_{i}$ is the total (corresponding) delay (including fueling and transport, etc.) for the $i$ th vehicle (see Huang and Ren (1997) for more details).

\subsection{Control law}

In [Huang and Ren (1998)], the proposed control law is given by:

$$
u_{i}(t)=k_{s}^{\prime} \delta_{i}(t)+k_{v}^{\prime} \dot{\delta}_{i}(t)+T_{L},
$$

where $k_{s}^{\prime}$ and $k_{v}^{\prime}$ are design constants. If one applies the control law (2) to the system (1), we shall obtain the following third order delay equation:

$$
\begin{aligned}
& \frac{d^{3}}{d t^{3}} \delta_{i}(t)=-\alpha \frac{d^{2}}{d t^{2}} \delta_{i}(t)-k_{s} \delta_{i}\left(t-\tau_{i}\right) \\
& -\left(k_{v}+\lambda k_{s}\right) \frac{d}{d t} \delta_{i}\left(t-\tau_{i}\right)-\lambda k_{v} \frac{d^{2}}{d t^{2}} \delta_{i}\left(t-\tau_{i}\right) \\
& +k_{s} \delta_{i-1}\left(t-\tau_{i-1}\right)+k_{v} \frac{d}{d t} \delta_{i-1}\left(t-\tau_{i-1}\right),
\end{aligned}
$$

where $k_{s}$ and $k_{v}$ are derived from $k_{s}^{\prime}$ and $k_{v}^{\prime}$ by an appropriate re-scaling. For the sake of simplicity, the corresponding computations are omitted (see Huang and Ren (1997)] and [Huang and Ren (1998)]).

\subsection{Problem formulation}

\subsubsection{Individual stability: Problem formulation A} basic control requirement for the overall system is the asymptotic stability of the $i$ th vehicle if the preceding, the $(i-1)$ th, is at steady-state (i.e. the spacing errors verify: $\delta_{i-1}=\dot{\delta}_{i-1}=0$ ). In this case, the system is described by:

$$
\begin{aligned}
& \frac{d^{3}}{d t^{3}} \delta_{i}(t)=-\alpha \frac{d^{2}}{d t^{2}} \delta_{i}(t)-k_{s} \delta_{i}\left(t-\tau_{i}\right) \\
& -\left(k_{v}+\lambda k_{s}\right) \frac{d}{d t} \delta_{i}\left(t-\tau_{i}\right)-\lambda k_{v} \frac{d^{2}}{d t^{2}} \delta_{i}\left(t-\tau_{i}\right) .
\end{aligned}
$$

Taking the Laplace transform, under zero initial conditions, we obtain a third-order transcendental equation of the form $\Gamma_{i}\left(s, \tau_{i}\right):=$

$$
\begin{aligned}
s^{3}+ & \alpha s^{2}+\left[\lambda k_{v} s^{2}+\left(k_{v}+\lambda k_{s}\right) s+k_{s}\right] e^{-\tau_{i} s} \\
= & Q(s)+P(s) e^{-s \tau}=0 .
\end{aligned}
$$

The individual vehicle stability is guaranteed if and only if $\Gamma$ has all its roots in the left half complex plane. This depends on the delay magnitude $\tau_{i}$. 
Then the problem of stability can be formulated as a research of parameters $\alpha, \lambda, k_{s}$ and $k_{v}$ such that this condition is ensured.

\subsubsection{Avoiding slinky effect: Problem formulation}

The second part of the multi-objective problem previously defined consist in controlling the slinky effect. The goal is to find sufficient conditions to guarantee that we avoid such a phenomenon. If we consider the system (3) and take Laplace transformation, we get $G(s)=\delta_{i}(s) / \delta_{i-1}(s)=$

$$
\frac{\left(k_{s}+s k_{v}\right) e^{-\tau_{i-1} s}}{\left(k_{s}+\left(k_{v}+\lambda k_{s}\right) s+\lambda k_{v} s^{2}\right) e^{-\tau_{i} s}+\alpha s^{2}+s^{3}} .
$$

We have no slinky-type effect if:

$$
|G(s)|=\left|\frac{\delta_{i}(j w)}{\delta_{i-1}(j w)}\right|<1
$$

for any $w>0$ (see [Ioannou and Chien (1993)], [Shiekholslam and Desoer (1993)], [Swaroop et al. (1994)]). Then the problem turns out in finding the set of parameters $\left(k_{s}, k_{v}\right)$ and the delays $\tau_{i}$ such that the stability of the system (4) is guaranteed and the condition (7) is satisfied (to avoid slinky-effect).

\section{MAIN RESULTS}

\subsection{Stability analysis}

Before proceeding further, we consider the case without delay. Analyzing the asymptotic stability of the closed-loop system free of delay turns out to check when the polynomial $\Gamma_{i}(s, 0)$, with $\tau_{i}=0$, is Hurwitz. Since $\alpha, k_{s}, k_{v}>0$, the third-order polynomial:

$$
s^{3}+\left(\alpha+\lambda k_{v}\right) s^{2}+\left(k_{v}+\lambda k_{s}\right) s+k_{s}=0
$$

is Hurwitz if and only if:

$$
\left(\alpha+\lambda k_{v}\right)\left(k_{v}+\lambda k_{s}\right)>k_{s},
$$

which is equivalent to

$$
\lambda k_{v}^{2}+\left(\alpha+\lambda^{2} k_{s}\right) k_{v}+(\alpha \lambda-1) k_{s}>0 .
$$

Note that a sufficient condition for (10) is:

$$
k_{v}>\frac{1-\alpha \lambda}{\lambda^{2}} \text {. }
$$

Define now by $\Omega$ the set of crossing frequencies, that is the set of reals $\omega>0$, such that $\pm j \omega$ is a solution of the characteristic equation (5). We have the following:

Proposition 1. Consider the characteristic equation (5) associated to the system (4). Then:

(a) the crossing frequency set $\Omega$ is not empty, and

(b) the system is asymptotically stable for all delays $\tau_{i} \in\left(0, \tau^{\star}\right)$ where $\tau^{\star}$ is defined by:

$$
\tau_{\star}=\frac{1}{w} \arccos \left(\frac{\alpha\left(k_{s}-\lambda k_{v} w^{2}\right) w^{2}+\left(k_{v}+\lambda k_{s}\right) w^{4}}{\left(k_{s}-\lambda k_{v} w^{2}\right)^{2}+\left(k_{v}+\lambda k_{s}\right)^{2} w^{2}}\right)(11)
$$

where $w$ is the unique element of $\Omega$.
The condition (a) above simply says that the corresponding system cannot be delay-independent asymptotically stable, and the condition $(b)$ above gives an explicit expression of the delay margin $\tau^{\star}$. In order to have a self-contained paper, a proof of the Proposition above is included in the Appendix. For a different proof, see, for instance, [?)].

\subsection{Avoiding slinky effects:}

Now, we consider the system (3). If we take Laplace transformation, then we obtain: $G(s)=\delta_{i}(s) / \delta_{i-1}(s)$

$$
=\frac{\left(k_{s}+s k_{v}\right) e^{-\tau_{i-1} s}}{\left(k_{s}+\left(k_{v}+\lambda k_{s}\right) s+\lambda k_{v} s^{2}\right) e^{-\tau_{i} s}+\alpha s^{2}+s^{3}} \text {. }
$$

There is no slinky effect if:

$$
|G(j w)|<1
$$

for any $w>0$. This condition can be rewritten as:

$$
A\left(w, \tau_{i}\right)(w)=w^{2} B\left(w, \tau_{i}\right) \geq 0,
$$

with

$$
\begin{aligned}
& B\left(w, \tau_{i}\right)(w)=w^{4}-2 \lambda k_{v} \sin \left(w \tau_{i}\right) w^{3}+ \\
& \left(\lambda^{2} k_{v}^{2}+\alpha^{2}+2\left(\alpha \lambda k_{v}-k_{v}-\lambda k_{s}\right) \cos \left(w \tau_{i}\right)\right) w^{2}+ \\
& 2\left(k_{s}-\alpha\left(k_{v}+\lambda k_{s}\right)\right) \sin \left(w \tau_{i}\right) w+ \\
& \lambda^{2} k_{s}^{2}-2 \alpha k_{s} \cos \left(w \tau_{i}\right),
\end{aligned}
$$

which should be satisfied for all $w \in \mathbb{R}$.

The objective is to define conditions on the parameters of the controller, in order to satisfy this constraint.

Consider first the case $\tau_{i}=0$. Then, we have:

$$
\begin{aligned}
& B(w, 0)=w^{4}+\left[\left(\lambda k_{v}+\alpha\right)^{2}-2\left(k_{v}+\lambda k_{s}\right)\right] w^{2} \\
& +\lambda^{2} k_{s}^{2}-2 \alpha k_{s} .
\end{aligned}
$$

A necessary condition for the positivity of $B(w, 0)$ is

$$
\lambda^{2} k_{s}^{2}-2 \alpha k_{s}>0
$$

which implies that:

$$
k_{s} \in\left(\frac{2 \alpha}{\lambda^{2}},+\infty\right)
$$

Under this condition, the positivity of $B(w, 0)$ is guaranteed if:

$$
\left[\left(\lambda k_{v}+\alpha\right)^{2}-2\left(k_{v}+\lambda k_{s}\right)\right]^{2} \leq 4\left(\lambda^{2} k_{s}^{2}-2 \alpha k_{s}\right) .
$$

which leads to:

$$
\begin{gathered}
-2 k_{s} \lambda \sqrt{1-\frac{2 \alpha}{\lambda^{2} k_{s}}} \leq\left(\lambda k_{v}+\alpha\right)^{2}-2\left(k_{v}+\lambda k_{s}\right) \\
\leq 2 k_{s} \lambda \sqrt{1-\frac{2 \alpha}{\lambda^{2} k_{s}}}
\end{gathered}
$$

In order to complete this analysis, we want to characterize the set of parameters $k_{v}$ guaranteeing the previous inequality under the constraint (17). 
If we consider first the right part of (19), which is equivalent to:

$\lambda^{2} k_{v}^{2}+2(\lambda \alpha-1) k_{v}+\alpha^{2}-2 \lambda k_{s}\left(1+\sqrt{1-\frac{2 \alpha}{\lambda^{2} k_{s}}}\right) \leq 0$, we can remark that if

$$
k_{s}>\max \left\{\frac{2 \alpha}{\lambda^{2}}, \frac{2 \alpha \lambda-1}{2 \lambda^{3}}\right\}
$$

then there exists at least one positive value $k_{v}$, such that the right part of (19) is satisfied. Moreover $k_{v}$ should satisfy:

$$
\max \left\{0, \frac{1-\alpha \lambda-\sqrt{\Delta_{1}}}{\lambda^{2}}\right\} \leq k_{v} \leq \frac{1-\alpha \lambda+\sqrt{\Delta_{1}}}{\lambda^{2}}
$$

where

$$
\Delta_{1}=1-2 \alpha \lambda+2 \lambda^{3} k_{s}\left(1+\sqrt{1-\frac{2 \alpha}{\lambda^{2} k_{s}}}\right) .
$$

The left inequality in (19) can be rewritten as:

$\lambda^{2} k_{v}^{2}+2(\lambda \alpha-1) k_{v}+\alpha^{2}-2 \lambda k_{s}\left(1-\sqrt{1-\frac{2 \alpha}{\lambda^{2} k_{s}}}\right) \geq 0$.

This leads to the following condition on $k_{v}$ :

$k_{v} \in\left(-\infty, \frac{1-\alpha \lambda-\sqrt{\Delta_{2}}}{\lambda^{2}}\right] \cup\left[\frac{1-\alpha \lambda+\sqrt{\Delta_{2}}}{\lambda^{2}},+\infty\right)$,

where

$$
\Delta_{2}=1-2 \alpha \lambda+2 \lambda^{3} k_{s}\left(1-\sqrt{1-\frac{2 \alpha}{\lambda^{2} k_{s}}}\right)
$$

is assumed to be positive. If $\Delta_{2}<0$, then the left part of (19) will be satisfied for all positive $k_{v}$.

Finally, using the conditions (21) and (22) function of the sign of $\Delta_{2}$, it follows that $k_{v}$ must be chosen in the intersection of the intervals defined by (21) and (22).

Now we analyze the sign of $B\left(w, \tau_{i}\right)$ when $\tau_{i} \geq 0$. We consider again the expression given in (14) of $B\left(w, \tau_{i}\right)$. For the terms involving $\cos \left(w \tau_{i}\right)$, we have:

$$
-2 \alpha k_{s} \cos \left(w \tau_{i}\right) \geq-2 \alpha k_{s}
$$

and

$2\left(\alpha \lambda k_{v}-k_{v}-\lambda k_{s}\right) \cos \left(w \tau_{i}\right) \geq-2\left|\alpha \lambda k_{v}-k_{v}-\lambda k_{s}\right|$.

Concerning the terms involving $\sin \left(w \tau_{i}\right)$, since $\sin \left(w \tau_{i}\right) \leq w \tau_{i}$ for $w>0$ then:

$$
-2 \lambda k_{v} \sin \left(w \tau_{i}\right) w^{3} \geq-2 \lambda k_{v} \tau_{i} w^{4} \geq-2 \lambda k_{v} \tau^{\star} w^{4},
$$
and

$$
\begin{aligned}
2\left(k_{s}-\alpha\left(k_{v}+\lambda k_{s}\right)\right) \sin \left(w \tau_{i}\right) w \\
\geq-2\left|k_{s}-\alpha\left(k_{v}+\lambda k_{s}\right)\right| \tau_{i} w^{2} \\
\geq-2\left|k_{s}-\alpha\left(k_{v}+\lambda k_{s}\right)\right| \tau^{\star} w^{2}
\end{aligned}
$$

Therefore,

$$
\begin{aligned}
& B\left(w, \tau_{i}\right) \geq\left(1-2 \lambda k_{v} \tau^{\star}\right) w^{4}+\left[\lambda^{2} k_{v}^{2}+\alpha^{2}\right. \\
& \left.-2\left|\alpha \lambda k_{v}-k_{v}-\lambda k_{s}\right|-2 \tau^{\star}\left|k_{s}-\alpha\left(k_{v}+\lambda k_{s}\right)\right|\right] w^{2} \\
& +\lambda^{2} k_{s}^{2}-2 \alpha k_{s} \\
& \geq\left(1-2 \lambda k_{v} \tau^{\star}\right) w^{4}+\left[\left(\lambda k_{v}-\alpha\right)^{2}-2 k_{v}-2 \lambda k_{s}\right. \\
& \left.-2 \tau^{\star} k_{s}-2 \tau^{\star} \alpha\left(k_{v}+\lambda k_{s}\right)\right] w^{2}+\lambda^{2} k_{s}^{2}-2 \alpha k_{s} \geq 0 .
\end{aligned}
$$

Let us set:

$C\left(w, \tau^{\star}\right)=\left(1-2 \lambda k_{v} \tau^{\star}\right) w^{4}+\left[\left(\lambda k_{v}-\alpha\right)^{2}-2 k_{v}\right.$ $\left.-2 \lambda k_{s}-2 \tau^{\star} k_{s}-2 \tau^{\star} \alpha\left(k_{v}+\lambda k_{s}\right)\right] w^{2}+\lambda^{2} k_{s}^{2}-2 \alpha k_{s}$

We suppose that:

$$
1-2 \lambda k_{v} \tau^{\star}>0 .
$$

Then the positivity of $C\left(w, \tau^{\star}\right)$ is ensured if (17) is satisfied and if we have:

$\left[\left(\lambda k_{v}-\alpha\right)^{2}-2 k_{v}-2 \lambda k_{s}-2 \tau^{\star} k_{s}\right.$ $\left.-2 \tau^{\star} \alpha\left(k_{v}+\lambda k_{s}\right)\right]^{2} \leq 4\left(1-2 \lambda k_{v} \tau^{\star}\right)\left(\lambda^{2} k_{s}^{2}-2 \alpha k_{s}\right)$.

This leads to the condition:

$$
\begin{gathered}
-2 k_{s} \lambda \sqrt{\left(1-\frac{2 \alpha}{\lambda^{2} k_{s}}\right)\left(1-2 \lambda k_{v} \tau^{\star}\right)} \leq \\
\left(\lambda k_{v}-\alpha\right)^{2}-2 k_{v}-2 \lambda k_{s}-2 \tau^{\star}\left(k_{s}+\alpha\left(k_{v}+\lambda k_{s}\right)\right) \\
\leq 2 k_{s} \lambda \sqrt{\left(1-\frac{2 \alpha}{\lambda^{2} k_{s}}\right)\left(1-2 \lambda k_{v} \tau^{\star}\right)}
\end{gathered}
$$

Now, we search to define the set of parameters $k_{v}$ which satisfy these inequalities. If we consider the right part of (25), which can be rewritten as:

$$
\begin{gathered}
\lambda^{2} k_{v}^{2}-2\left(1+\alpha \lambda+\alpha \tau^{\star}\right) k_{v}+\alpha^{2}-2 \tau^{\star}\left(k_{s}+\alpha \lambda k_{s}\right) \\
-2 \lambda k_{s}\left(1+\sqrt{\left(1-\frac{2 \alpha}{\lambda^{2} k_{s}}\right)\left(1-2 \lambda k_{v} \tau^{\star}\right)}\right) \leq 0
\end{gathered}
$$

with $k_{v}$ under the square root. Since $1-2 \lambda k_{v} \tau^{\star} \leq 1$ and $1-\frac{2 \alpha}{\lambda^{2} k_{s}} \leq 1$ then

$$
\begin{aligned}
& \lambda^{2} k_{v}^{2}-2\left(1+\alpha \lambda+\alpha \tau^{\star}\right) k_{v}+\alpha^{2}-2 \tau^{\star}\left(k_{s}+\alpha \lambda k_{s}\right) \\
& -2 \lambda k_{s}\left(1+\sqrt{\left(1-\frac{2 \alpha}{\lambda^{2} k_{s}}\right)\left(1-2 \lambda k_{v} \tau^{\star}\right)}\right) \\
& \leq \lambda^{2} k_{v}^{2}-2\left(1+\alpha \lambda+\alpha \tau^{\star}\right) k_{v}+\alpha^{2}-2 \tau^{\star}\left(k_{s}+\alpha \lambda k_{s}\right) \\
& -2 \lambda k_{s}\left(1+\left(1-2 \lambda k_{v} \tau^{\star}\right)\left(1-\frac{2 \alpha}{\lambda^{2} k_{s}}\right)\right)
\end{aligned}
$$

Thus, if we can find $k_{v}$ such that:

$$
\begin{aligned}
& \lambda^{2} k_{v}^{2}-2\left(1+\alpha \lambda+5 \alpha \tau^{\star}-2 \tau^{\star} \lambda^{2} k_{s}\right) k_{v} \\
& +\alpha^{2}-2 \tau^{\star}(1+\alpha \lambda) k_{s}-4 \lambda k_{s}+\frac{4 \alpha}{\lambda} \leq 0
\end{aligned}
$$

then the right part of (25), would be satisfied.

A necessary condition to guarantee this previous condition is to have:

$$
\begin{aligned}
\Delta_{1, \tau^{\star}}= & \left(1+\alpha \lambda+5 \alpha \tau^{\star}-2 \tau^{\star} \lambda^{2} k_{s}\right)^{2} \\
& -\lambda^{2}\left(\alpha^{2}-2 \tau^{\star}(1+\alpha \lambda) k_{s}-4 \lambda k_{s}+\frac{4 \alpha}{\lambda}\right) \\
& \geq 0
\end{aligned}
$$

and then under this condition, we choose $k_{v}$ as follows: 


$$
\max \left\{0, \frac{a_{1}-\sqrt{\Delta_{1, \tau^{\star}}}}{\lambda^{2}}\right\} \leq k_{v} \leq \frac{a_{1}+\sqrt{\Delta_{1, \tau^{\star}}}}{\lambda^{2}},
$$

where $a_{1}=1+\alpha \lambda+5 \alpha \tau^{\star}-2 \tau^{\star} \lambda^{2} k_{s}$.

We can remark that (29) can be rewritten as:

$$
\begin{aligned}
& 4 \tau^{\star^{2}} \lambda^{4} k_{s}^{2}+2 \lambda^{2}\left(\tau^{\star}(1+\alpha \lambda)+2 \lambda\right. \\
& \left.-2 \tau^{\star}\left(1+\alpha \lambda+5 \alpha \tau^{\star}\right)\right) k_{s} \\
& +\left(1+5 \alpha \tau^{\star}\right)^{2}+10 \alpha^{2} \tau^{\star} \lambda-2 \alpha \lambda \geq 0 .
\end{aligned}
$$

Note that this last inequality leads to the following condition on $k_{s}$ :

$$
k_{s} \in\left(-\infty, \xi_{1}\right] \bigcup\left[\xi_{2},+\infty\right)
$$

where:

$$
\begin{aligned}
& \xi_{1}=\frac{2 \tau^{\star}\left(1+\alpha \lambda+5 \alpha \tau^{\star}\right) \lambda^{2}-2 \lambda^{3}-\tau^{\star}(1+\alpha \lambda) \lambda^{2}-\sqrt{\overline{\Delta_{1, \tau^{\star}}}}}{4 \tau^{\star^{2}} \lambda^{4}} \\
& \xi_{2}=\frac{2 \tau^{\star}\left(1+\alpha \lambda+5 \alpha \tau^{\star}\right) \lambda^{2}-2 \lambda^{3}-\tau^{\star}(1+\alpha \lambda) \lambda^{2}+\sqrt{\overline{\Delta_{1, \tau^{\star}}}}}{4 \tau^{\star^{2}} \lambda^{4}},
\end{aligned}
$$

where

$$
\begin{gathered}
\overline{\Delta_{1, \tau^{\star}}}=\lambda^{4}\left(\tau^{\star}(1+\alpha \lambda)+2 \lambda-2 \tau^{\star}\left(1+\alpha \lambda+5 \alpha \tau^{\star}\right)\right)^{2} \\
-4 \tau^{\star^{2}} \lambda^{4}\left[\left(1+5 \alpha \tau^{\star}\right)^{2}+10 \alpha^{2} \tau^{\star} \lambda-2 \alpha \lambda\right]
\end{gathered}
$$

which is supposed to be positive. If it is not the case, then the condition (29) is verified for all $k_{s} \geq 0$.

We consider now the left part of (25), which can be rewritten as:

$$
\begin{aligned}
0 & \leq \lambda^{2} k_{v}^{2}-2\left(1+\alpha \lambda+\alpha \tau^{\star}\right) k_{v}+\alpha^{2}-2 \tau^{\star}\left(k_{s}+\alpha \lambda k_{s}\right) \\
& -2 \lambda k_{s}\left(1-\sqrt{\left(1-\frac{2 \alpha}{\lambda^{2} k_{s}}\right)\left(1-2 \lambda k_{v} \tau^{\star}\right)}\right) .
\end{aligned}
$$

Proceeding as above, we have:

$$
\begin{aligned}
& \lambda^{2} k_{v}^{2}-2\left(1+\alpha \lambda+\alpha \tau^{\star}\right) k_{v}+\alpha^{2}-2 \tau^{\star}\left(k_{s}+\alpha \lambda k_{s}\right) \\
& \quad-2 \lambda k_{s}\left(1-\left(1-2 \lambda k_{v} \tau^{\star}\right)\left(1-\frac{2 \alpha}{\lambda^{2} k_{s}}\right)\right) \\
& \leq \lambda^{2} k_{v}^{2}-2\left(1+\alpha \lambda+\alpha \tau^{\star}\right) k_{v}+\alpha^{2}-2 \tau^{\star}\left(k_{s}+\alpha \lambda k_{s}\right) \\
& -2 \lambda k_{s}\left(1-\sqrt{\left(1-\frac{2 \alpha}{\lambda^{2} k_{s}}\right)\left(1-2 \lambda k_{v} \tau^{\star}\right)}\right) .
\end{aligned}
$$

If there exists $k_{v}$ such that:

$$
\begin{aligned}
0 \leq & \lambda^{2} k_{v}^{2}-2\left(1+\alpha \lambda+\alpha \tau^{\star}\right. \\
& \left.+2 \tau^{\star} \lambda^{2} k_{s}\left(1-\frac{2 \alpha}{\lambda^{2} k_{s}}\right)\right) k_{v} \\
& +\alpha^{2}-2 \tau^{\star}\left(k_{s}+\alpha \lambda k_{s}\right)-2 \lambda k_{s}\left(1-\left(1-\frac{2 \alpha}{\lambda^{2} k_{s}}\right)\right),
\end{aligned}
$$

then the left part of (25), will be verified. This inequality can be simplified as:

$$
\begin{gathered}
0 \leq \lambda^{2} k_{v}^{2}-2\left(1+\alpha \lambda-3 \alpha \tau^{\star}+2 \tau^{\star} \lambda^{2} k_{s}\right) k_{v} \\
+\alpha^{2}-2 \tau^{\star}(1+\alpha \lambda) k_{s}-\frac{4 \alpha}{\lambda} .
\end{gathered}
$$

This is satisfied for all $k_{v}$ such that:

$$
\begin{aligned}
& k_{v} \in\left(-\infty, \frac{1+\alpha \lambda-3 \alpha \tau^{\star}+2 \tau^{\star} \lambda^{2} k_{s}-\sqrt{\Delta_{2, \tau^{\star}}}}{\lambda^{2}}\right] \\
& \bigcup\left[\frac{1+\alpha \lambda-3 \alpha \tau^{\star}+2 \tau^{\star} \lambda^{2} k_{s}+\sqrt{\Delta_{2, \tau^{\star}}}}{\lambda^{2}},+\infty\right),
\end{aligned}
$$

where

$$
\begin{aligned}
\Delta_{2, \tau^{\star}} & =\left(1+\alpha \lambda-3 \alpha \tau^{\star}+2 \tau^{\star} \lambda^{2} k_{s}\right)^{2} \\
& -\lambda^{2}\left(\alpha^{2}-2 \tau^{\star}(1+\alpha \lambda) k_{s}-\frac{4 \alpha}{\lambda}\right)
\end{aligned}
$$

is supposed to be positive. If this quantity is negative, then the inequality (34) and by consequence (32), would be satisfied for all $k_{v} \geq 0$. The positivity of $\Delta_{2, \tau^{\star}}$ can be rewritten as:

$$
\begin{aligned}
& 4 \tau^{\star^{2}} \lambda^{4} k_{s}^{2}+6 \lambda^{2} \tau^{\star}\left[1+\alpha-2 \alpha \tau^{\star}\right] k_{s} \\
& +\left(1-3 \alpha \tau^{\star}\right)^{2}+6 \alpha \lambda\left(1-\alpha \tau^{\star}\right) \geq 0
\end{aligned}
$$

which leads to the condition on $k_{s}$ given by:

$$
\begin{gathered}
k_{s} \in\left(-\infty, \frac{\left.3 \lambda^{2} \tau^{\star}\left(2 \alpha \tau^{\star}-1-\alpha\right)-\sqrt{\overline{\Delta_{2, \tau^{\star}}}}\right]}{4 \lambda^{4} \tau^{\star^{2}}}\right] \\
\bigcup\left[\frac{\left.3 \lambda^{2} \tau^{\star}\left(2 \alpha \tau^{\star}-1-\alpha\right)+\sqrt{\overline{\Delta_{2, \tau^{\star}}}},+\infty\right)}{4 \lambda^{4} \tau^{\star^{2}}},\right.
\end{gathered}
$$

if $\overline{\Delta_{2, \tau^{\star}}}$ defined by:

$$
\begin{aligned}
\overline{\Delta_{2, \tau^{\star}}} & =9 \lambda^{4} \tau^{\star^{2}}\left[1+\alpha-2 \alpha \tau^{\star}\right]^{2} \\
& -4 \lambda^{4} \tau^{\star^{2}}\left[\left(1-3 \alpha \tau^{\star}\right)^{2}+6 \alpha \lambda\left(1-\alpha \tau^{\star}\right)\right]
\end{aligned}
$$

is positive.

It is clear that if $\overline{\Delta_{2, \tau^{*}}}$ is negative, then the positivity of $\Delta_{2, \tau^{\star}}$ would be satisfied for all $k_{s} \geq 0$. Now the hypothesis of negativity of $\Delta_{2, \tau^{*}}$, which would imply that the left part of (25) is satisfied for all $k_{v}$ positive, turns out to write that:

$$
\begin{aligned}
& 4 \tau^{\star^{2}} \lambda^{4} k_{s}^{2}+6 \lambda^{2} \tau^{\star}\left[1+\alpha-2 \alpha \tau^{\star}\right] k_{s} \\
& +\left(1-3 \alpha \tau^{\star}\right)^{2}+6 \alpha \lambda\left(1-\alpha \tau^{\star}\right) \leq 0,
\end{aligned}
$$

which is satisfied for

$$
\begin{array}{r}
\max \left\{0, \frac{3 \lambda^{2} \tau^{\star}\left(2 \alpha \tau^{\star}-1-\alpha\right)-\sqrt{\overline{\Delta_{2, \tau^{\star}}}}}{4 \lambda^{4} \tau^{\star^{2}}}\right\} \leq k_{s} \\
\leq \frac{3 \lambda^{2} \tau^{\star}\left(2 \alpha \tau^{\star}-1-\alpha\right)+\sqrt{\overline{\Delta_{2, \tau^{\star}}}}}{4 \lambda^{4} \tau^{\star^{2}}},
\end{array}
$$

where $\overline{\Delta_{2, \tau^{\star}}}$ is assumed to be positive.

In conclusion, the determination of the parameters $k_{v}$ and $k_{s}$ guaranteeing that (25) is satisfied, can be summarized for the right part of (25), by the choice of $k_{v}$ in the interval defined by (30) under the necessary condition that $\Delta_{1, \tau^{\star}}$ is positive. And for the left part 
of (25), we can choose any $k_{v}>0$ or $k_{v}$ in the interval defined by (36), according to the sign of $\Delta_{2, \tau^{\star}}$.

We can note that $\Delta_{1, \tau^{\star}}$ and $\Delta_{2, \tau^{\star}}$ are function of $k_{s}$. Their sign are conditioned by the sign of $\overline{\Delta_{1, \tau^{\star}}}$ and $\overline{\Delta_{2, \tau^{\star}}}$.

\section{ILLUSTRATIVE EXAMPLES}

Consider the system (4) with the parameters $\alpha=$ $5, \lambda=0,85, k_{s}=14,8$ and $k_{v}=2,41$. This example has been considered in [Huang and Ren (1998)], where the authors obtained a delay bound $\tau^{*}<0.041$. By using Proposition 1, we obtain the optimal delay margin equal to $\tau^{\star}=0.405$. The system (4) is then asymptotically stable for all delays $\tau<0.405$. We arrive to the same conclusion by using the Matlab package DDE-BIFTOOL (bifurcation analysis of delay differential equations), (see [Engelborghs et al. (2002)], [?)]) to represent the rightmost roots of the characteristic equation. Indeed, if we choose the limit value of the delay $\tau=0.405$ then we can observe that rightmost roots of the characteristic equation are on the imaginary axis.

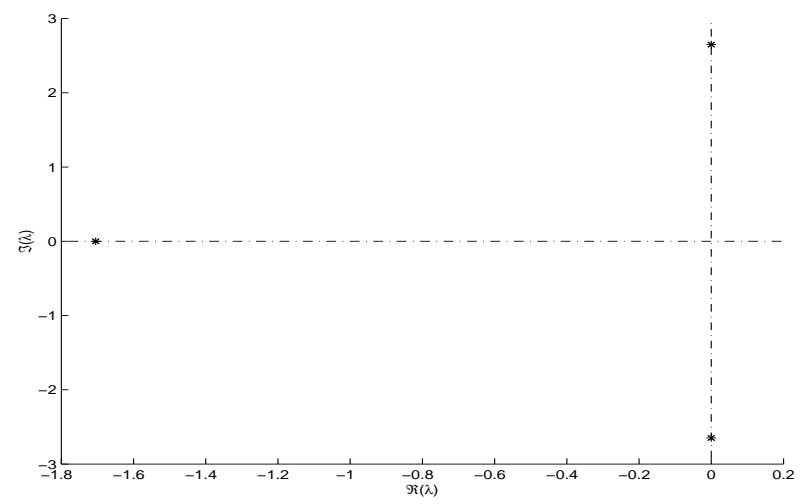

Fig. 2. Roots of the characteristic equation for $\tau=$ 0.405

Now, if we consider the second part of the multiobjective problem, we can remark that the conditions to avoid slinky-effect given by [Huang and Ren (1998)] enable us to choose $\tau^{\star}=0.1637$. Therefore combined with the condition of stability that we established, we can take a delay $\tau \leq \min (0.1637,0.405)=$ 0.1637 which remains better than the bound $\tau^{*}<$ 0.041 proposed in [Huang and Ren (1998)].

However, if we consider the conditions that we established for avoiding slinky-effect, then the choice of parameters: $\alpha=5, \lambda=0,85, k_{s}=14,8$ and $k_{v}=2,41$ doesn't fulfill the conditions that we established. More precisely, the necessary condition (16) is satisfied, but the condition to avoid slinky effect is not satisfied by this set of parameters since the condition (18) is not verified. Moreover, for delay $\tau>0$, (example $\tau^{\star}=0.1637$ ), the assumption (23) is satisfied but not the condition (25). Thus, the condition to avoid slinky

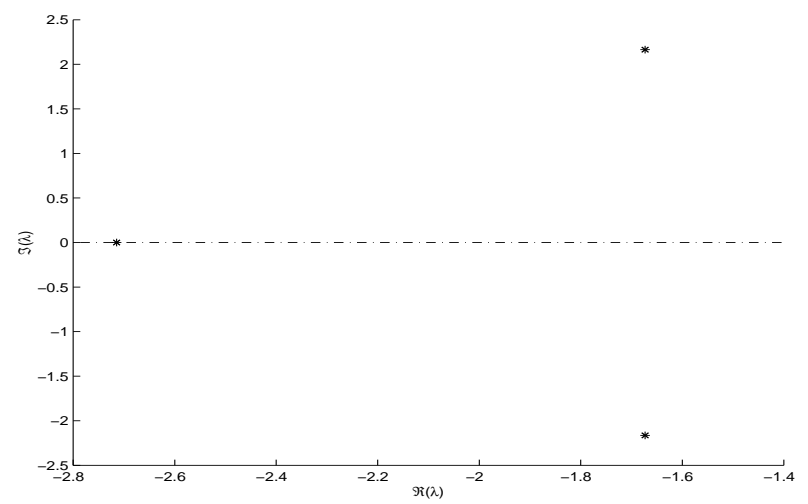

Fig. 3. Roots of the characteristic equation for $\tau=$ 0.1637

effects when the delay is different from zero is no satisfied. However, there exists set of parameters such that the stability can be guaranteed and the condition to avoid slinky type effects proposed in this paper are fulfilled. If we choose $\alpha=5, \lambda=1, k_{s}=19$ and $k_{v}=0.12$, then by Proposition 1, the delay bound is $\tau^{*}=0.215$, and in order to have no slinky effects we just have to restrict this bound to $\tau=0.0504$. Moreover, for this set of parameters, the conditions to avoid slinky effect established by [Huang and Ren (1998)] are not satisfied. In fact, the condition (c) of Theorem 2 is not verified.

\section{CONCLUSIONS}

In this paper, we have considered the problem of vehicle following control system. For a given controller structure, we have developed conditions guaranteeing the individual stability of each vehicle of the platoon, and the derived conditions depend on the size of the delay. Moreover, we considered the problem of slinkyeffect phenomenon, and we proposed sufficient conditions to avoid it. We have given an explicit characterization of some sets of controller parameters which solve the problem.

\section{REFERENCES}

Burnham, G.O., Seo, J. and Bekey, G.A. (1974). Identification of human drivers models in car following, IEEE Transactions on Automatic Control, vol. 19, pp. 911-915.

Cooke, K. L. and van den Driessche, P. (1986). On zeroes of some transcendental equations. in Funkcialaj Ekvacioj, vol. 29, pp. 77-90.

Engelborghs, K., Luzyanina, T. and Samaey, G. (2001). DDE-BIFTOOL v. 2.00: a Matlab package for bifurcation analysis of delay differential equations, Technical Report TW-330, Departement of Computer Science, K.U.Leuven, Belgium.

Engelborghs, K., Luzyanina, T. and Roose, R. (2002) Numerical bifurcation analysis of delay differential equations using DDE-BIFTOOL, ACM Trans. Math. Softw., vol. 28, pp. 1-21. 
El'sgol'ts, L.E. and Norkin, S.B. (1973). Introduction to the theory and applications of differential equations with deviating arguments (Academic Press: New York).

Gu, K., Kharitonov, V.L. and Chen, J. (2003). Stability of time-delay systems (Birkhauser: Boston).

Hale, J. K. and Verduyn Lunel, S. M. (1993). Introduction to Functional Differential Equations (Applied Math. Sciences, 99, Springer-Verlag, New York).

Huang, S. and Ren, W. (1997). Design of vehicle following control systems with actuator delays, in International Journal of Systems Science, vol. 28, pp. 145-151.

Huang, S. and Ren, W. (1998). Autonomous Intelligent Cruise Control with Actuator Delays, in Journal of Intelligent and Robotic Systems, vol. 23, pp. 27-43.

Huang, S. and Ren, W. (1999). Automatic vehicle following with integrated throttle and brake control, in International Journal of Control, vol. 72, pp. 7583.

Ioannou, P.A. and Chien, C.C. (1993). Autonomous intelligent cruise control, in IEEE Transactions on Vehicular Technology, vol. 18, pp. 657-672.

Michiels, W. (2002). Stability and stabilization of time-delay systems (Ph.D. Thesis, Katholieke Universiteit Leuven, Belgium, May 2002).

Niculescu, S.-I. (2001). Delay effects on stability. A robust control approach (Springer-Verlag: Heidelberg, LNCIS, vol. 269).

Pasino, K. (1995). A mixture of intelligent and conventional control methods may be the best way to implement autonomous systems, IEEE Spectrum, vol. 32, pp. 55-62.

Sipahi, R. (2005). Cluster Treatment of Characteristic Roots, CTCR, A Unique Methodology for the Complete Stability Robustness Analysis of Linear Time Invariant Multiple Time Delay Systems Against Delay Uncertainties (Ph.D. Thesis, University of Connecticut, Mechanical Engineering Department, August 2005).

Sipahi, R. and Niculescu, S.-I. (2007). Deterministic time-delayed traffic flow models: A survey. in (F.M. ATAY, ED.), Springer-Verlag (to appear).

Shiekholslam, S. and Desoer, C.A. (1993). Longitudinal control of a platoon of vehicles with no communication of lead vehicle information: a system study, in IEEE Transactions on Vehicular Technology, vol. 42, pp. 546-554.

Swaroop, D., Hedrick, J.K., Chien, C.C. and Ioannou, P.A. (1994). A comparison of spacing and headway control laws for automatically controlled vehicles, Vehicle System Dynamics, vol. 23, pp. 597-625.

Varaiya, P. (1993). Smart cars on smart roads: problems of control, in IEEE Transactions on Automatic Control, vol. 38, pp. 195-207.
Appendix A. PROOF OF PROPOSITION 1

(a) Straightforward. Assume by contradiction that the delay-independent stability holds. As discussed in [Niculescu (2001)], a necessary condition for delayindependent stability is the Hurwitz stability of $Q$, and this is not the case.

(b) Since the system free of delay is asymptotically stable, the conclusion of (a) leads to the existence of a delay margin $\tau^{\star}$, such that the system is asymptotically stable for all delays $\tau \in\left[0, \tau^{\star}\right)$. Furthermore at $\tau=\tau^{\star}$, the characteristic equation (5) has at least one root $s=j w$ on the imaginary axis, with $w \in \Omega$ (crossing frequency). Since

$$
\frac{P(j w)}{Q(j w)}=-e^{-j w \tau}=-\cos (w \tau)+j \sin (w \tau)
$$

this implies that:

$$
\cos (w \tau)=-\Re\left(\frac{P(j w)}{Q(j w)}\right) .
$$

We compute the right hand side of this equation with:

$$
\begin{aligned}
\frac{P(j w)}{Q(j w)} & =-\frac{\alpha\left(k_{s}-\lambda k_{v} w^{2}\right) w^{2}+\left(k_{v}+\lambda k_{s}\right) w^{4}}{\left(k_{s}-\lambda k_{v} w^{2}\right)^{2}+\left(k_{v}+\lambda k_{s}\right)^{2} w^{2}} \\
& -\frac{j\left(k_{s}-\lambda k_{v} w^{2}\right) w^{3}-j \alpha\left(k_{v}+\lambda k_{s}\right) w^{3}}{\left(k_{s}-\lambda k_{v} w^{2}\right)^{2}+\left(k_{v}+\lambda k_{s}\right)^{2} w^{2}}
\end{aligned}
$$

Therefore,

$\tau^{\star}=\frac{1}{w} \arccos \left(\frac{\alpha\left(k_{s}-\lambda k_{v} w^{2}\right) w^{2}+\left(k_{v}+\lambda k_{s}\right) w^{4}}{\left(k_{s}-\lambda k_{v} w^{2}\right)^{2}+\left(k_{v}+\lambda k_{s}\right)^{2} w^{2}}\right)$,

where $w$ is a crossing frequency.

In the sequel, we explicitly determinate the expression of the crossing frequencies by solving the equation:

$$
w^{6}+\left(\alpha^{2}-\lambda^{2} k_{v}^{2}\right) w^{4}-\left(k_{v}^{2}+\lambda^{2} k_{s}^{2}\right) w^{2}-k_{s}^{2}=0 .
$$

For this equation in $w^{2}$, we have one real solution (and two complex roots) or three real roots. We have to analyze their sign to consider only the positive candidates.

If we denote by $r_{i},(i=1 \ldots 3)$, the roots of the equation, we know that they are solutions of:

$$
x^{3}-S x^{2}+\Pi_{2} x-\Pi_{3}=0,
$$

where $S=\sum_{i=1}^{3} r_{i}, \quad \Pi_{2}=\prod_{i \neq j \in\{1 \ldots 3\}} r_{i} r_{j}, \quad \Pi_{3}=\prod_{i \in\{1 \ldots 3\}} r_{i}$.

Since $\Pi_{3}=k_{s}^{2}>0$, if we have only one real root (the others are complex and conjugate), this root is positive and if we have three real roots, we have one positive root and two real roots with the same sign. In the latter case, we only take into account only the case where the three real roots are positive. Moreover, with $\Pi_{2}=-\left(k_{v}^{2}+\lambda^{2} k_{s}^{2}\right)<0$, we can remark that we cannot have three positive real roots. Finally, we can have only one positive real root (square of the crossing frequency). Now we apply the method of Cardan to 
define the form of this crossing frequency. We can establish that if:

$$
\begin{aligned}
& \left(\alpha^{4}+\lambda^{2}\left(\lambda^{2} k_{v}^{4}+3 k_{s}^{2}-2 \alpha^{2} k_{v}^{2}\right)+3 k_{v}^{2}\right)^{3} \\
& <\frac{1}{4}\left(\left(\alpha^{2}-\lambda^{2} k_{v}^{2}\right)\left[2\left(\alpha^{2}-\lambda^{2} k_{v}^{2}\right)+9\left(\lambda^{2} k_{s}^{2}+k_{v}^{2}\right)\right]-27 k_{s}^{2}\right)^{2},
\end{aligned}
$$

then the crossing frequency is of the form:

$$
w_{f}=\sqrt{\left(-\frac{w_{1}}{54}\right)^{\frac{1}{3}}+\left(-\frac{w_{2}}{54}\right)^{\frac{1}{3}}-\frac{\alpha^{2}-\lambda^{2} k_{v}^{2}}{3}}
$$

where

$$
w_{1}=\gamma_{1}+\sqrt{\zeta_{1}} \text { and } w_{2}=\gamma_{1}-\sqrt{\zeta_{1}},(\text { A.6) }
$$

with

$\gamma_{1}=\left(\left(\alpha^{2}-\lambda^{2} k_{v}^{2}\right)\left[2\left(\alpha^{2}-\lambda^{2} k_{v}^{2}\right)^{2}+9\left(\lambda^{2} k_{s}^{2}+k_{v}^{2}\right)\right]-27 k_{s}^{2}\right)$,

and

$$
\zeta_{1}=\gamma_{1}^{2}-4\left(\left(\alpha^{2}-\lambda^{2} k_{v}^{2}\right)^{2}+3\left(\lambda^{2} k_{s}^{2}+k_{v}^{2}\right)\right)^{3}
$$

If

$$
\begin{aligned}
& \left(\alpha^{4}+\lambda^{2}\left(\lambda^{2} k_{v}^{4}+3 k_{s}^{2}-2 \alpha^{2} k_{v}^{2}\right)+3 k_{v}^{2}\right)^{3} \\
& >\frac{1}{4}\left(\left(\alpha^{2}-\lambda^{2} k_{v}^{2}\right)\left[2\left(\alpha^{2}-\lambda^{2} k_{v}^{2}\right)+9\left(\lambda^{2} k_{s}^{2}+k_{v}^{2}\right)\right]-27 k_{s}^{2}\right)^{2},
\end{aligned}
$$

then it is of the form :

$$
w_{f}=\sqrt{\left(-\frac{\tilde{w}_{1}}{54}\right)^{\frac{1}{3}}+\left(-\frac{\tilde{w}_{2}}{54}\right)^{\frac{1}{3}}-\frac{\alpha^{2}-\lambda^{2} k_{v}^{2}}{3}}
$$

where

$$
\tilde{w}_{1}=\gamma_{1}+j \sqrt{-\zeta_{1}} \text { and } \tilde{w}_{2}=\gamma_{1}-j \sqrt{-\zeta_{1}}(\mathrm{~A} .8)
$$

